\title{
A Simulation Environment for Maxillofacial Surgery Including Soft Tissue Implications
}

\author{
Filip Schutyser ${ }^{1}$, Johan Van Cleynenbreugel ${ }^{1}$, Joseph Schoenaers $^{2}$, \\ Guy Marchal ${ }^{1}$, and Paul Suetens ${ }^{1}$
}

1 Laboratory for Medical Image Computing (ESAT and Radiology) K.U.Leuven

2 Departement of Stomatology and Maxillofacial Surgery

University Hospitals of Leuven, Herestraat 49, B-3000 Leuven, Belgium

Filip.Schutyser@uz.kuleuven.ac.be

\begin{abstract}
This paper describes work in progress in the area of planning and prediction of maxillofacial surgery. We focus on true 3D problems which cannot be addressed by conventional 2D cephalometric radiographs. Examples are bilateral repositioning of the zygoma's or treating facial asymmetries. The environment being developed arose from the need of patients for prediction of their expected post-op outlook and from the need of surgeons for better support to reach the desired result. Our simulation environment adheres to a scene-based approach in which CT image-derived visualizations and additional 3D photographs (showing the face surface and its natural complexion) are co-presented and manipulated. Tools for non-planar osteotomy are included. Repositioning of bone fragments is made possible. Furthermore, a voxel displacement formalism is presented to account for soft tissue implications. Combining these simulation tools, a qualitative prediction of the facial outlook is obtained. Results are shown for a case of hypoplastic zygoma repositioning.
\end{abstract}

Keywords Image guided therapy, maxillofacial surgery simulation, osteotomy, soft tissue modelling

\section{Introduction}

The human face plays a key role in interpersonal relationships. Indeed, facial outlook is of utmost importance in recognizing and remembering another person. Consequently, people are very sensitive to changes in this outlook. In the case of facial malformations (caused by e.g. car-accidents, tumors, congenital dysmorphologies, ... ) this concern is even more severe as maxillofacial surgery is often required. The important question "What will I look like after surgery ?" is not always easy to answer, however.

To predict expected surgical results, an image based simulation system can be considered. However the success of such an approach depends on how it deals with requirements specific to maxillofacial prediction:

- Natural facial perception is 3D. Standard cephalometric images usually show lateral projections only, which is certainly not the facial impression obtained by looking at one-selve's in a mirror. 
- The repositioning of bone fragments after osteotomy is often a real 3D problem. Typical examples are bilateral repositioning of the zygomas or reconstruction of facial symmetry.

- To a patient, it is not only important to predict the post-operative shape of his/her face, but also how this shape will look in combination with his/her natural complexion.

This paper describes the current state of an image based system that is meant to cope with the above requirements. With this system, surgical actions on underlying bony structures are simulated. Their implications on surrounding soft tissue (the shape of the skin) are calculated. The expected result of the surgery is shown to the patient in a convincing and realistic manner.

Section 2 discusses current planning procedures and related research work for maxillofacial surgery including soft tissue implications. In section 3 our developments are explained. Concluding remarks finish the paper in section 4 .

\section{Background}

A maxillofacial intervention is normally planned by means of lateral X-ray images. Using these so-called cephalograms, distances (based on cephalometric landmarks) are measured and compared to their normal values. In this way a surgeon derives a surgical plan while the patient is provided with the expected new contour of the face. This image-on-film based planning has been extended towards 2D image based computer systems, some of them even capable of introducing video images [1]. However, predictability is limited due to the lack to explore 3D movement and its consequences. Planning methods based on 3D images may open new possibilities to handle the requirements mentioned in the previous section.

One 3D attempt is the use of tangible skull models produced by e.g. stereolithograpy [2]. These models make realistic bone surgery simulation possible. However, disadvantages are a large extra cost of the model, a considerable production time and a loss of information concerning soft tissues. Instead of tactile models, 3D software planning systems are another line of work. Ideally, such systems would include capabilities for osteotomy simulation, cephalometric reference data for guidance during repositioning, and finally ways for modelling soft tissue deformation. In practice, no single system integrating these features has been reported on:

1. Osteotomy simulation for craniofacial surgery is surveyed by Vannier et al. [3]. More recent examples are the work of Altobelli et al. [4], Keeve et al. [5] and Bohner et al. [8]. In general this work is restricted to planar cuts.

2. Cutting et al. [21] describe an early method to introduce 3D cephalometric constraints. However, only a limited transfer of well-known cephalometric landmarks from lateral and frontal 2D X-ray analysis towards 3D image analysis is possible (see also [9]). Altobelli et al. [4] apply interactive repositioning using cephalometric and anthropometric databases. This previous 
work mentioned the paucity of normative 3D data as a significant obstacle. Mollard et al. [10] recently report on an integration of 3D cephalometry into a simulator for orthognatic surgery.

3. For modelling soft tissue deformation, a number of (intuitive) physics based, models are described in the literature. In all these approaches, the derivation of tissue material properties (e.g. generalised constant of Hooke) from image data is treated harshly. On the other hand, applying such models in a simulation environment usually means to trade-off interactivity against accuracy. Mass-spring models represent soft tissue as a collection of point masses connected by (nonlinear) springs in a lattice structure. E.g. in [5] mass-spring modeling of the skin tissue is used resulting in a model that can be updated at interactive speeds, but represents a significant approximation. In continuum models, deformation is described as a function of external forces and of the tissue's material properties. When such models are solved by finite element methods (FEM), physically realistic simulations can be provided, however at the expense of loosing interactivity, e.g. [11]. To speed up FEM, several assumptions (and thus reduced flexibility) can be made to perform part of the calculations in a pre-processing step $[12,13]$. Voxel-based methods, focusing on fast respons time without sacrificing to much realism, are explored by Gibson et al., [6,7]. Such methods combine a kinematic model (ChainMail) determining the coarse motion of a discretized deformable object to a dynamic model (mass-spring model) to refine the final shape of the object.

\section{Methods}

\subsection{D Environment}

Our current simulation system expands on the ideas we have previously described in [14] and applied to other surgical disciplines in [15] and [16]. Basically we adhere to a scene-based approach in which image derived visualizations (MPR, surfaces, volume renderings) and additional 3D structures (external to the medical image volume) are co-presented and manipulated. As an example of the latter - typical for maxillofacial applications -, see figure 1. The underlying graphics environment is OpenInventor [17].

Primarily we employ CT data. It is reasonably easy to segment bone structures and soft tissues of interest from this source. To provide the patient with a realistic view of the natural complexion of his/her face, we also acquire a 3D photograph. Currently we use an "active" 3D system (projecting a pattern on the face and recovering $3 \mathrm{D}$ from the deformation of the pattern on the $2 \mathrm{D}$ picture), see [18]. The surface thus obtained is registered with the skin surface extracted from the CT using point/surface matching algorithms like ICP [19] or a 3D extension of RPM [20]. A result was shown in figure 1 already. 

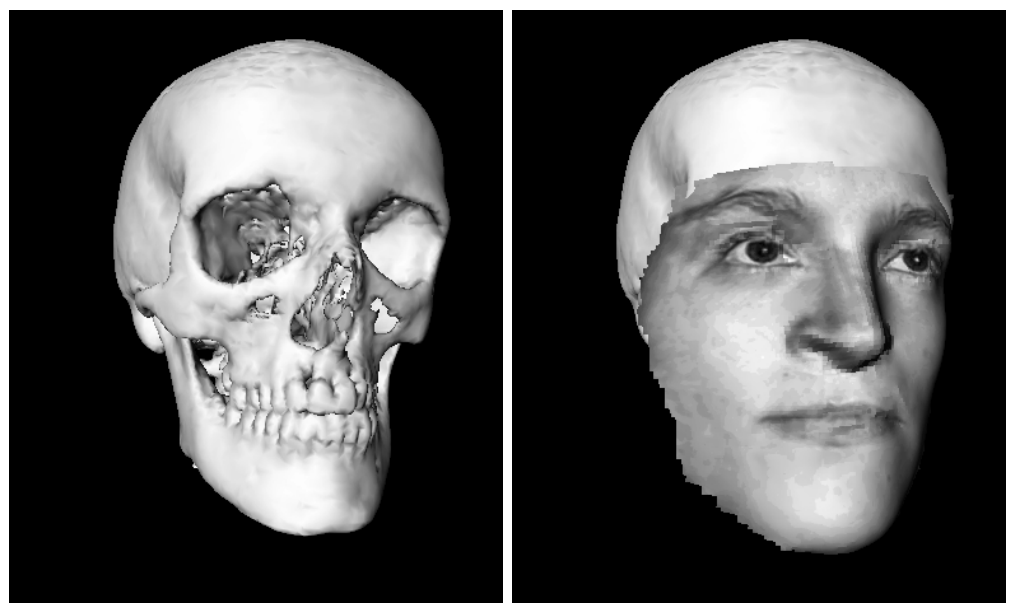

Fig. 1. Our surgical simulation environment presents anatomical objects in a 3D scene. The bony structures derived from CT images, can be co-presented with arbitrarily chosen multiplanar reslices and for example so-called 3D photographs.

\subsection{Osteotomy Planning: Cutting and Repositioning of Bone}

To simulate osteotomy, first a cutting blade is defined on a surface representation of the bone, then the cutting action is performed, and finally repositioning of the bone fragments is made possible.

The definition of the cutting blade is a two step procedure. First, the surgeon draws a trajectory on the bone surface (using the workstation's mouse). While drawing, this surface can be rotated and translated to have an optimal sight on the structure of interest (figure 2). Real time manipulation is possible due to graphics features such as overlay planes and depth buffers. Second, the surgeon specifies the depth along the cutting trajectory. This depth is edited by means of a manipulator (see figure 2 or by means of a 3D cursor (3D mouse) in combination with stereoviewing (shutter glasses)). Once the trajectory is thus extended into a cutting blade, the actual cutting action is performed on the volume data itself (by labeling voxels adjacent to the blade and recalculating bone surfaces, see figure 3).

Repositioning is the final step in every osteotomy simulation. However it is not sufficient to provide general tools to freely rotate and translate resectioned bone fragments in 3D space. Extra guidance should be introduced. In [15] we have already shown that in this way the repeatability of a planning procedure can be increased and the planning time be decreased. It will be clear that $3 \mathrm{D}$ cephalometry is the best provider of medical knowledge in this context. However as pointed out in section 2, no reliable sources are yet to be found, leaving this part of our simulation environment as work in progress. 

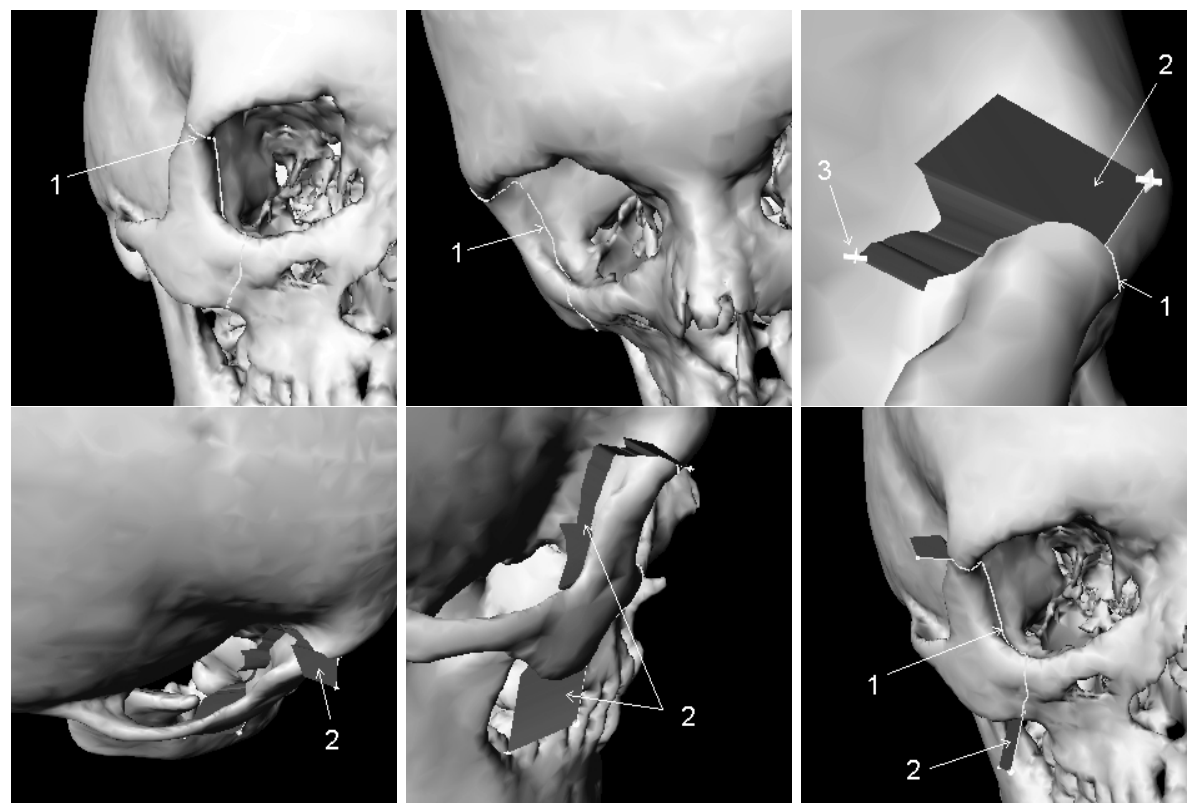

Fig. 2. In this example, an osteotomy trajectory (1) is being drawn on a zygoma. Due to its complex anatomy, the outlined trajectory is a true $3 \mathrm{D}$ curve. The cutting blade (2) is specified as shown in the upper right image. The manipulator (3) is used to change the direction and extent of the depth of the blade along the cutting trajectory. The final result is depicted in the bottom row.
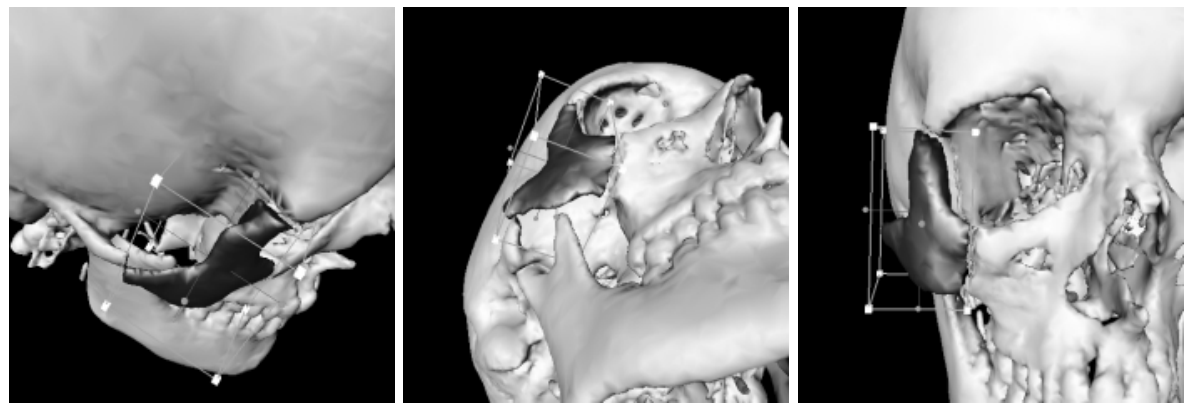

Fig. 3. As a result of applying the cutting blade, the zygoma is resectioned (darker part) from the skull. A manipulator is attached to it in order to allow repositioning. 


\subsection{Soft Tissue Modelling}

While FEM methods are promising, current FEM formulations and software implementations are not practical for real-time modeling of complex geometry and detailed interior structure. However, complex system behaviour can result from applying simple behaviour patterns to a large number of elements. Based on this observation, we state a new formalism for voxel-based tissue displacement induced by sectioning and repositioning of bone.

Indeed, as a result of our osteotomy and repositioning simulation, the displacement of one or more bone tissue part(s) $B$ is known. Hence, in the preoperative image volume a displacement vector $\Delta(v)$ is known for every voxel $v \in B$. We want to extend this partially defined displacement vector field $\Delta$ towards a new vector field $\bar{\Delta}$ that maps the entire pre-operative image volume into a "to be expected" postoperative image volume. Of course, $\forall v \in B, \bar{\Delta}(v)=\Delta(v)$. Furthermore, for our application of maxillofacial surgery, we are mainly interested in the behaviour of $\bar{\Delta}$ on voxels "between" bone and skin.

So how can $\Delta(v)$ be extended towards $\bar{\Delta}(v)$ on a voxel level? We model $\bar{\Delta}(v)$ in a voxel $v \notin B$ by taking into account three factors (sources of influence). First, suppose $N_{v}=\left\{w_{1}, \cdots, w_{n}\right\}$ is a set of voxels neighbouring $v$. Then it is reasonable to expect that $\bar{\Delta}$ is behaving in $v$ in a way consistent with its behaviour in $N_{v}$. Stated otherwise, the displacement in $v$ depends on the displacement of its neigbouring voxels. Second, as $v$ belongs to a given tissue type, also material properties, say $M(v)$ will contribute to the expected displacement. Finally, the way $v$ interacts with its neighbouring voxels ties the previous sources together. We denote this interaction as the function $\Phi$, resulting in the relationship

$$
\bar{\Delta}(v)=\Phi\left(\bar{\Delta}\left(w_{1}\right), \cdots, \bar{\Delta}\left(w_{n}\right), M(v)\right)
$$

This equation will generally be used in an iterative way starting from the known values of $\bar{\Delta}$ in the voxels belonging to $B$. In this way the equation drives a relaxation scheme. Of course a variety of extended displacement fields can be obtained by varying $N_{v}, M$ and $\Phi$.

To illustrate this approach, assume the three sources of influence have the following meaning:

1. Let $N_{v}$ the set of six facet neighbours of $v$.

2. Assume that for a voxel $v$ belonging to soft tissue, $M(v)$ represents a displacement attenuation factor typical for the soft tissue.

3. The interaction of $v$ with its neigbouring voxels is expressed as taking the maximum of the neighbouring displacement vectors and attuenuating it by the factor $M(v)$.

For each iteration and for each voxel $v$ belonging to the soft tissue of interest, $\bar{\Delta}(v)$ is calculated as stated by source 3 . Convergence of iteration is assumed when the difference in the displacement field between two iteration steps is smaller than a given $\epsilon$.

A result is shown in figure 4. Initially the zygoma was repositioned according to the expertise of the surgeon. The original 3D photograph has been locally displaced by apptying the extended displacement field to the skin surface. 

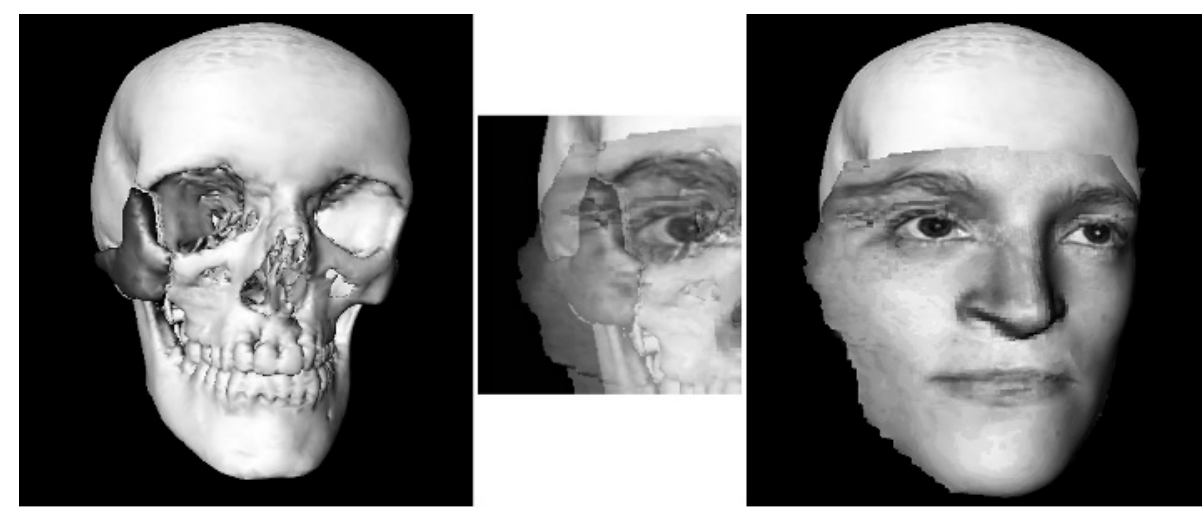

Fig. 4. On the left, a hypoplastic zygoma is repositioned to approximate the ideal sizes of the skull. Using our approach of extending the displacement field known on the bone fragment towards the surrounding soft tissues, the skin surface is locally deformed.

\section{Conclusion}

This paper describes a 3D environment for simulation and prediction of true 3D problems in maxillofacial surgery. Examples are treating asymmetries, bilateral repositioning of the zygoma's, .... These problems comprise three parts: cutting of the skull, repositioning of the bone fragments and modelling the soft skin tissue to predict the expected face after surgery.

Concerning osteotomies, we are able to cut the bone according to free-hand drawn 3D trajectories and cutting blades. Uptill now, we allow free 3D repositioning of the bone fragments (three dimensions of freedom for rotation and three dimensions of freedom for rotation). Giving the surgeon extra guidance to reposition the bone fragments using 3D cephalometry can be an interesting tool speeding up the simulation process. However, as explained, analysis based on 3D cephalometry needs further investigations.

We introduced a general voxel-based formalism for predicting the face after surgery. We instantiated the formalism in a concrete implementation. Comparing our approach to the voxel-based methods of Gibson $[6,7]$ it will be clear that the latter requires adaptions and generalisations for the application of maxillo-facial surgery: at least two different kind of tissue need to be introduced and attention needs to given to displacements induced by osteotomy and repositioning. Also the ChainMail's important property of visiting only once needs reconsideration in the case of different types of tissue and the presence of non-convex structures.

The focus of further research will be on refinement of the soft tissue deformation model on incorporation of 3D cephalometric analysis and on validation of this approach towards clinical application. 


\section{Acknowledgments}

The work discussed here belongs, partly to the grant GOA/99/05 (VHS+ Variability in Human Shape and Speech) of the K.U.Leuven Research Council, and partly to the EU-funded Brite Euram III PISA project (nr. BRPR CT97 0378). Partners in the latter are Materialise NV, Belgium; Philips Medical Systems BV, the Netherlands: ICS-AD; DePuy International Ltd, UK; Ceka NV, Belgium; K.U. Leuven, Belgium: ESAT/Radiology \& Div. Biomechanics; University of Leeds, UK: Research School of Medicine.

\section{References}

1. G. Schultes, Q. Gaggl, H. Kärcher: Accuracy of Cephalometric and Video Imaging Program Dentofacial Planner Plus in Orthognathic Surgical Planning. Computer Aided Surgery, Vol. 3, Nr. 3, p 108-114, 19981211

2. J. Kragskov, S. Sindet-Petersen, C. Gyldensted, K.L. Jensen: A comparison of threedimensional computed tomography scans and stereolithographic models for evaluation of craniofacial anomalies. Journal of Oral and Maxillofacial Surgery, 54:4, p 402-411, April 19961211

3. M. Vannier, J. Marsh, A. Tsiaras: Craniofacial Surgical Planning and Evaluation with Computers. Computer Integrated Surgery (eds. Taylor, Lavallee, Burdea, Mosges) MIT press 1996, p 673-678 1211

4. D. Altobelli, R. Kikinis, J. Mulliken, H. Cline, W. Lorensen, F. Jolesz ComputerAssisted Three-Dimensional Planning in Craniofacial Surgery Plastic and Reconstructive Surgery, 1993, vol 92, p 576-585 1211

5. E. Keeve, S. Girod, B. Girod: Craniofacial Surgery Simulation. Proceedings of the 4th International Conference on Visualisation in Biomedical Computing VBC'96, p 541-546 1211, 1212

6. S. Gibson 3D Chainmail: a fast algorithm for deforming volumetric objects. Proc. Symposium on Interactive 3D Graphics, ACM SIGGRAPH, p 149-154 1212, 1216

7. S. Gibson, C. Fyock, E. Grimson, T. Kanade, R. Kikinis, H. Lauer, N. McKenzie, A. Mor, S. Nakajima, H. Ohkami, R. Osborne, J. Samosky, A. Sawada Volumetric modeling for surgical simulation. Medical Image Analysis, 2(2), p 121-132 1212, 1216

8. P. Bohner, C. Holler, S. Hassfeld Operation Planning in Craniomaxillofacial Surgery Computer Aided Surgery, 1997, Vol 2(3/4), p 153-161 1211

9. R. Fuhrmann, H. Feifel, A. Schnappauf, P. Diedrich: Integration of ThreeDimensional Cephalometry and 3D-Skull Models in Combined Orthodontic/Surgical Treatment Planning. Journal of Otofacial Orthopedics, 57:1, p 32-45, February 1996 1211

10. B. Mollard, S. Lavallée, G. Bettega: Computer Assisted Orthognatic Surgery. Proc. MICCAI98, Lect. Notes in Computer Science, 1496, 1998, p 21-28 1212

11. S.D. Kirby, B. Wang, C.W.S. To, H.B. Lampe: Nonlinear, Three-Dimensional Finite-Element Model of Skin Biomechanics Journal of Otolaryngology, 27:3, p 153160, June 19981212

12. Fast finite elements for surgery simulation. Medicine Meets Virtual Reality V, 1997 1212

13. Real-time volumetric deformable models for surgery simulation using finite elements and condensation. Proc. Eurographics, vol. 15, p 57-66, 19961212 
14. J. Van Cleynenbreugel, K. Verstreken, G. Marchal, P. Suetens: A flexible environment for image guided virtual surgery planning. Proc. VBC96, Lect. Notes in Computer Science, 1131, 1996, p 501-510 1212

15. F. Schutyser, J. Van Cleynenbreugel, V. Vander Poorten, P. Delaere, G. Marchal, P. Suetens: An experimental image guided surgery simulator for hemicricolaryngectomy and reconstrunction by tracheal autotransplantation. Proc. MICCAI98, Lect. Notes in Computer Science, 1496, 1998, p 919-925 1212, 1213

16. K. Verstreken, J. Van Cleynenbreugel, K. Martens, G. Marchal, D. van Steenberghe, P. Suetens: An image-guided Planning System for Endosseous Oral Implants. IEEE Transactions on Medical Imaging Vol 17, no 5, pp. 842-852, October 19981212

17. J. Werneke: The Inventor Mentor, Programming Object-Oriented 3D Graphics with OpenInventor Addison-Wesley, release 2, 19941212

18. ShapeSnatcher, www.eyetronics.com 1212

19. Paul J. Besl and Neil D. McKay: A Method for Registration of 3-D Shapes. IEEE Trans. on Pattern Analysis and Machine Intelligence, 14:2, p 239-256, 19921212

20. Anand Rangarajan, Haili Chui, Eric Mjolsness, Suguna Pappu, Lila Davachi, Patricia Goldman-Rakic, James Duncan: A robust point-matching algorithm for autoradiograph alignment. Medical Image Analysis, Vol. 1, Nr. 4, p 379-398, 1997 1212

21. C. Cutting, F. Bookstein, B. Grayson, L. Felligham, J. McCarthy ThreeDimensional Computer-Assisted Design of Craniofacial Surgical Procedures: Optimization and Interaction with Cephalometric and CT-Based Models. Plastic and Reconstructive Surgery, 1986, vol 77, p 877-885 1211 